\title{
Laparoscopic Retrieval of Intra-Abdominal Foreign Bodies
}

\author{
Siva Gounder and Jeremy Tan* \\ Sir Charles Gairdner Hospital, Australia
}

Submission: February 2, 2017; Published: February 23, 2017

*Corresponding author: Jeremy Tan, Sir Charles Gairdner Hospital, G Block, Hospital Ave, Nedlands WA 6009, Perth, Australia, Email: jthtan1@gmail.com

\begin{abstract}
Foreign bodies causing intra abdominal infection is not a common occurrence. There are multiple reports of laparoscopic retrieval of intra-luminal and extra-luminal foreign bodies within the peritoneal cavity. However, to date, there have not been any reports of a laparoscopic extra-peritoneal approach to retrieve a foreign body lodged in the pelvic side wall. We also describe a challenging second case of laparoscopic retrieval of a fish bone stuck within the wall of the stomach which was perforating into the liver, causing a liver abscess. In both cases available radiological and endoscopic facilities were utilized to accurately localize and assist removal of the foreign body successfully.
\end{abstract}

\section{Introduction}

Intra-abdominal foreign bodies can be either secondary to ingestion or trans-abdominal wall migration. They pose a challenge in diagnosis as well as management. Most ingested foreign bodies pass through the gastrointestinal tract uneventfully [1-7]. Only a small percentage are symptomatic either causing perforation or obstruction. Similarly, foreign bodies can migrate into the abdominal cavity through the abdominal wall and come to lie in the intra-peritoneal or extraperitoneal space. In this report we describe two cases where both intra-peritoneal and extra-peritoneal foreign bodies were removed laparoscopically.

\section{Case Reports}

\section{Case 1}

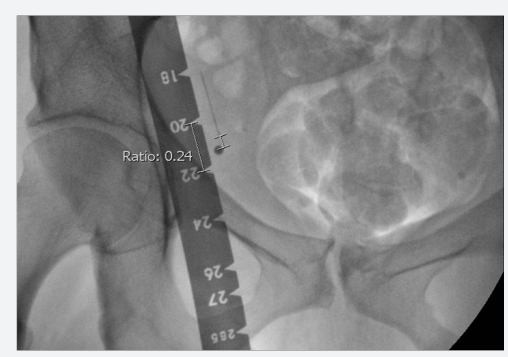

Figure 1: Intra-operative $\mathrm{X}$-ray showing needle in right groin area.

The patient was a 50 year old intravenous drug user who was in the habit of injecting amphetamines and had a known history of Hepatitis C infection. One month prior to presentation, the whole of a 23 gauge needle snapped off subcutaneously following an injection into the right femoral vein. The patient presented to a regional hospital where her right groin region was explored twice under local anaesthetic. The first attempt was without image intensification and the second time was with image intensification (Figure 1). Both attempts were unsuccessful in retrieving the needle.

The patient was then discharged but about three weeks later, presented with cellulitis and a groin wound which was discharging pus. She was placed on intravenous antibiotics and transferred to our hospital, where the vascular surgeons explored her again in search of the needle. They opened the peritoneal cavity as well but were unable to locate the needle. She then went on to have a CT guided insertion of a hookwire from the skin just over the right superior pubic ramus to the needle, which was located in the extra-peritoneal fat, just medial to the external iliac vessels (Figure 2).

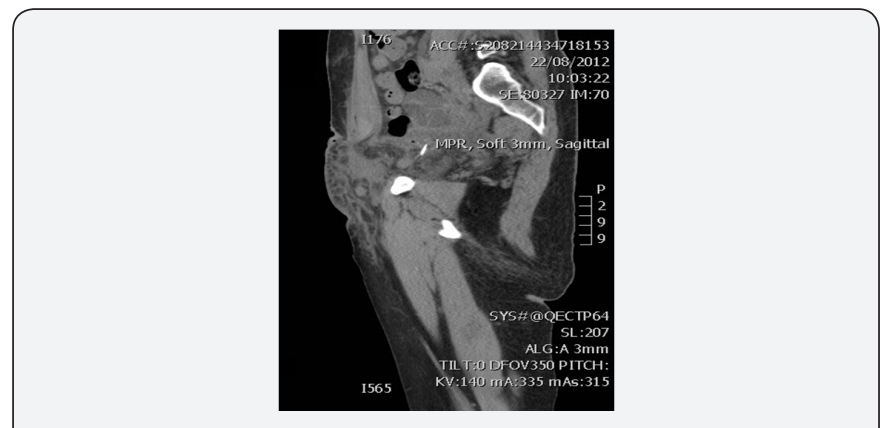

Figure 2: Sagittal CT scan showing needle in right groin region. 
She was then referred to our general surgical service for retrieval of the foreign body. It was felt that the most expedient approach would be a laparoscopic extra-peritoneal approach, similar to the approach for a totally extra-peritoneal (TEP) inguinal hernia repair. We waited two days for the peritoneum in the region to heal and we also did discuss with the patient the option of leaving the needle in-situ, but given that she had already experienced infective complications, she was not keen for this.

At surgery, the right rectus sheath was entered just to the right and inferior to the umbilicus. The dissection balloon was not used for fear of dislodging the hookwire. Blunt finger dissection for about $3 \mathrm{~cm}$, was done initially and the first $12 \mathrm{~mm}$ port was inserted. Gas insufflation to $12 \mathrm{mmHg}$ was commenced once the laparoscope was inserted and we verified that we were in the right plane. Further blunt dissection with the tip of the scope was done towards the midline and a $5 \mathrm{~mm}$ port was placed midway between the umbilicus and the superior pubic ramus.

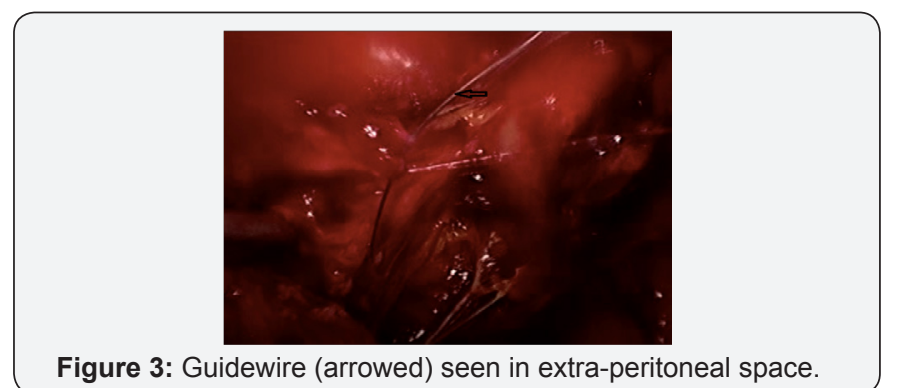

Through this port further blunt dissection with a blunttipped instrument was performed laterally and caudally until the hookwire was found in the extra-peritoneal plane (Figure 3). A second $5 \mathrm{~mm}$ port was placed laterally, at the level of the first $5 \mathrm{~mm}$ port, ensuring good triangulation of the working ports and the camera port. Sutures could be seen in the peritoneum where the vascular surgeons had explored two days previously.

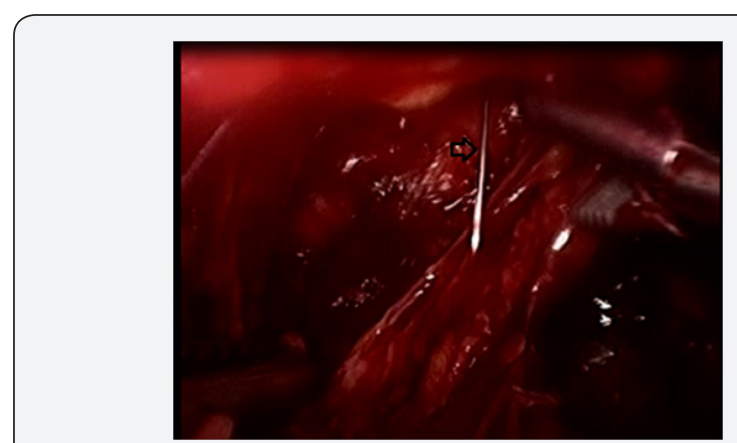

Figure 4: Intra-operative photo showing needle (arrowed) in extra-peritoneal space. Guidewire has been removed.

Further gradual blunt dissection with two graspers was performed following the hookwire, deeper into the pelvis in the extra-peritoneal plane. This was continued until the needle popped into view (Figure 4). It was grasped and retrieved via the right lateral $5 \mathrm{~mm}$ port. There was no obvious breach of the peritoneum. The procedure was then converted to routine intra-peritoneal laparoscopy via the umbilical port to inspect the bowel in the pelvis to confirm that everything was intact. The bowel appeared intact and there was no breach seen in the peritoneum.

The fascia at the umbilical port site was closed with ' 0 ' Vicryl and the skin wounds were closed with a subcuticular suture. The patient made an uneventful recovery and was discharged on post-operative day three. The delay was mainly secondary due to the fact that she lived in the country and still required daily dressing to her open groin wound.

\section{Case 2}

The patient was a 40 year old part time lawyer who had presented to our institution three weeks prior with RUQ pain. $\mathrm{U} / \mathrm{S}$ of the abdomen showed sludge in the gall bladder, but HIDA scan was negative. She was discharged, with a follow-up clinic appointment to discuss whether or not she should have an elective laparoscopic cholecystectomy.

She represented prior to the outpatient appointment with fevers, epigastric and right upper quadrant pain. A working diagnosis of cholecystitis was made. However, a repeat ultrasound showed a likely $6 \mathrm{~cm}$ diameter liver abscess in segment 3 of the liver.

As she was already scheduled for theatre and it was felt that her pain 3 weeks ago might have been biliary colic, a decision was made to proceed with laparoscopic cholecystectomy and laparoscopic drainage of the liver abscess at the same time. Intraoperatively, the gall bladder appeared macroscopically normal. It was removed without performance of a cholangiogram. The left lobe of the liver was swollen and had a yellow tinge to it. The abscess was drained using an airway needle and a 19 Fr Blake's drain was placed into the cavity.

The patient progressed well, and was placed on intravenous antibiotics in the form of Tazocin (piperacillin and tazobactam), initially and then benzylpenicillin when the sensitivities were known. The bacteria grown were Streptococcus aginosus. However, on Day 5 post-op, she started getting fevers again to 38.5 degrees $\mathrm{C}$ and a CT scan showed that the drain had fallen out of the cavity and that the liver abscess had re-accumulated (Figure 5).

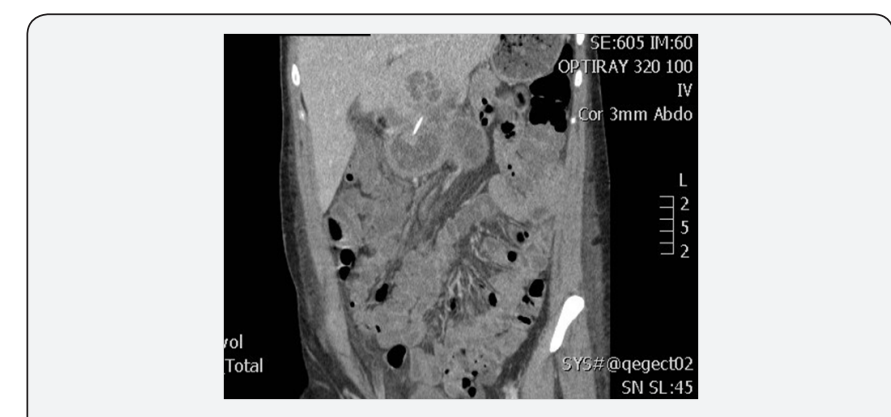

Figure 5: Coronal CT scan showing fishbone and associated liver abscess. 
She thus went on to have ultrasound-guided percutaneous drainage of the recurrent abscess. At this time, the radiologist noted an echogenic structure in the wall of the stomach and with correlation with the CT scan and by speaking to the patient, elucidated that it was a fish bone lodged between the stomach and the liver. The patient had hurriedly eaten a whole fish about 6 weeks prior to presentation.

As such, the patient returned to theatre the next day for laparoscopic removal of the fish bone. The chronic tract between the left lobe of liver and the stomach was disconnected, but no fish bone was seen. After some aimless and fruitless blunt exploration of both the posterior surface of the abscess and the perigastric fat around the antrum, laparoscopic intra-operative ultrasound was performed and this found the fish bone within the wall of the stomach. On table gastroscopy was also performed and although there was a visible bulge with a central punctum visible in the antrum anteriorly, no actual bone could be seen. A long airway needle was then passed percutaneously, through the peritoneal cavity and through the anterior wall of the stomach until the tip could be seen in the stomach in the location of the lump, to be used as a marker. However, as the stomach was deflated and the gastroscope withdrawn, the fish bone popped into view laparoscopically and was retrieved. It was sent for $\mathrm{MC}+\mathrm{S}$ but did not grow anything.

Omentum was tacked onto the stomach to form a barrier between the stomach and the liver, and the existing peritoneal drain was left nearby. The percutaneous drain was left in-situ, in the abscess. It was noted that the abscess was very firm and fibrotic at this stage and not at all fluctuant. She made a good recovery from there and was discharged home 4 days later on intravenous ceftriaxone $2 \mathrm{~g}$ daily and oral metronidazole. The peritoneal drain was removed prior to discharge. The pigtail percutaneous drain was removed about a week later. At followup, the patient remains well.

\section{Discussion}

Ingested foreign body causing intra-abdominal abscess is a well recognized complication. Migration of foreign body from one site to another as in the first case is also a known cause of intra-abdominal abscess $[1,2,4]$. Foreign body migration through the gastrointestinal tract does not necessarily cause peritonitis [3]. Also, as in our patient, a high proportion of patients don't remember ingesting the offending foreign body $[6,7]$.

Many reports of laparoscopic removal of foreign body has been described in the literature [4,8-10]. However, we have not found any other cases in the literature documenting a laparoscopic extraperitoneal approach to removal of foreign bodies. The first patient had Hepatitis $\mathrm{C}$ and although an open extraperitoneal approach could have been used, it was felt that the chance of a needlestick injury was greatly reduced using the laparoscopic extra-peritoneal approach. Also, post-operative morbidity to the patient was reduced with a laparoscopic approach.
These two cases thus demonstrate removal of challenging foreign bodies causing infection. In the first case, a laparoscopic extraperitoneal approach with hookwire guidance was used, and in the second case, laparoscopic intra-operative ultrasound and gastroscopy were employed. One of the key lessons is that it helps to have some sort of percutaneously inserted marker leading to the foreign body to help in its localisation. In the first case a hookwire (identical to the ones used for wide local excision in breast surgery) was used and in the second case an airway needle was used.

In the second case, we needed laparoscopic ultrasound and gastroscopy to help localize the foreign body, but we feel that it was the airway needle, skewering the anterior wall of the stomach, next to the fish bone, which acted as a fulcrum to help lever the fish bone to the visceral peritoneal surface when the stomach was deflated which helped in its retrieval.

The fall back plan was to explore along the track of the airway needle until the fish bone was located, but this was not necessary. Both patients benefitted from a minimally invasive approach and have recovered well. It is a pity however, that both needed more than one surgical procedure each. To some extent, lack of forward planning and inadequate pre-operative imaging and preparation led to both patients requiring more than one operation to solve their problem. Nevertheless, both cases illustrate the importance of a multi-disciplinary team approach, use of adjunct imaging, and use of specialized localization methods in achieving a good outcome for the patients.

\section{Conclusion}

Intra-abdominal foreign bodies can prevent a challenging clinical scenario. Technically, they can be difficult to localize and retrieve. Their removal is important if there is associated infection. Image-guided percutaneous localization preoperatively or intra-operatively can be useful.

\section{References}

1. Santos SA, Alberto SC, Cruz E, Pires E, Figueira T, et al. (2007) Hepatic abscess induced by foreign body: Case report and literature review. World Journal of Gastroenterology 13(9): 1466-1470.

2. Chen HK, Kuo JR, Uen YH, Sun DP (2011) Liver abscess secondary to fish bone migration from the duodenum 81(3): 206.

3. Kendra K, Walter P, Yi-Horng L (2012) Transluminal migration of ingested foreign body without peritonitis. Journal of Pediatric Surgery 47(4): 788-791.

4. Dominguez S, Wildhaber BE, Spadola L, Mehrak AD, Chardot C (2009) Laparoscopic extraction of intra hepatic foreign body after transduodenal migration in a child. Journal of Pediatric Surgery 44(11): E17-E20.

5. Aarabi S, Stephenson J, Christie DL, Javid PJ (2012) Noningested intraperitoneal foreign body causing chronic abdominal pain: a role for laparoscopy in diagnosis. Journal of Pediatric Surgery 47(20: E15-E17.

6. Rodríguez-Hermosa JI, Codina-Cazador A, Sirvent JM, Martín A, Gironès J, et al. (2008) Surgically treated perforations of gastrointestinal tract caused by ingested foreign bodies. Colorectal Disease 10(7): 701-707.

7. Goh BK, Chow PK, Quah HM, Ong HS, Eu KW, et al. (2006) Perforation of 
gastrointestinal tract secondary to ingestion of foreign bodies. World Surg (30): 372-377

8. Rodrigues D, Perez NE, Hammer PM, John DW (2006) Laparoscopic removal of a retained intra-abdominal ribbon malleable retractor after 14 years. J Laparoendosc Adv Surg Tech 16: 369-371.
9. Le Mandat-Schultz A, Bonnard A, Belarbi N, Aigrain Y, Lagausie PD (2003) Intrahepatic foreign body laparoscopic extraction. Surg Endosc 17(11): 1849

10. Omejc M (2002) Laparoscopic removal of an ingested pin migrating into the liver. Surg Endosc 16(3): 537.

Your next submission with Juniper Publishers will reach you the below assets

- Quality Editorial service

- Swift Peer Review

- Reprints availability

- E-prints Service

- Manuscript Podcast for convenient understanding

- Global attainment for your research

- Manuscript accessibility in different formats

( Pdf, E-pub, Full Text, Audio)

- Unceasing customer service

Track the below URL for one-step submission https://juniperpublishers.com/online-submission.php 